\title{
Learnart : Drawing Environment using Convolutional Neural Networks
}

\author{
Duraimurugan.N, Manoj Kumar.B, Malini.C, Kowsalya.R
}

\begin{abstract}
Simulating human consciousness and emotions is still the realm of science fiction. The future of neural networks will not exist in tasks to simulate realization. Nowadays, further learning is based on training the machine to recognize the target that might be image or words using datasets. The project is based on convolutional neural networks and weak artificial intelligence. It uses Back Propagation algorithm. It acts as a smart drawing platform for children. Predefined datasets will be framed when it is developed so that users cannot alter it. Users will be the one who draw and checks its efficiency. Suggestion will be displayed if it is right or wrong. The drawings will be shapes and real time objects. The platform gets trained by all these datasets and recognizes the object. The drawing is stored as pixels pattern and checks with the previous data and finds how much percentage does the current drawing is matching with the previous drawings. By the help of this application, children can develop basic single object drawing.
\end{abstract}

Keywords - Artificial Intelligence, Deep Learning, Convolutional Neural Networks, Back Propagation

\section{INTRODUCTION}

Human beings have innovated the ability of computer systems with their divergent overall fields, reducing size in terms of time and their increasing swiftness. Artificial Intelligence pursues building the computers or machines as brainy as human beings. Artificial Intelligence is cultivating its path for future automation. Every domain including education and entertainment is getting influenced by Artificial Intelligence. Edutainment is getting upgraded by much application involving artificial intelligence.

In this paper, we propose a drawing environment for children who can practice single object drawings utilizing it. To analyse the visual imagery Convolutional neural networks is been most commonly applied. Datasets fed are used to analyse the input and produce the output. The purpose for developing this application is to support beginners.

\section{RELATED WORKS}

Several works have influenced our proposed project. Drawing curves in mid-air [6] with fingers is a fundamental task with applications to 3D sketching, geometric modelling, handwriting recognition, and authentication. Mid-air curve input is most commonly accomplished through explicit user input; akin to click-and-drag, the user may use a hand posture (e.g. pinch) or a button-press on an instrumented controller to express the intention to start and stop sketching. Positioning attribute of this paper helped in our

Revised Manuscript Received on July 10, 2019.

Duraimurugan.N, India

Manojkumar.B, India

Malini.C, India

Kowsalya.R,India project. Paper [2] presents the HabilisDraw interface, explores the benefits of a desktop metaphor that closely mimics the behaviour of tools and objects in a twodimensional drawing environment, and argues for the applicability of the system's fundamental principles for improving interface usability in the future.

The other system [4] receives a motif data set and a user's sketch image, and returns advice to the user. They developed and experimented a prototype system limited to treat a basic motif and principal advice. As a result, the validity of the proposed system was confirmed. A symmetrical feature selection criterion is first introduced to select robust matches of local key points between two images. The Mallows distance is then adopted to combine multiple cues from statistics of local matches. This experiment [7] confirm that SSL based approach not only boost classification performance but also improve robustness of the learned category model using only simple local key point features. This paper [1] describes Smart Canvas, an intelligent desk system that allows a user to perform freehand drawing on a desk or similar surface with gestures.

\section{MOTIVATION}

Nowadays, everything is becoming smart such home, car, education, etc. If we glance smart education field, there won't be much drawing-based application for kids based on neural networks. There is only little smart drawing application developed when compared to the applications developed for other purposes. Especially for edutainment not so many applications have craved its identity in market The early stage children who are in need to develop their drawing skills do not have adequate options for the search.

\section{MODULES DESIGN}

LearnART is the module-based action application. The main motive is to develop the drawing skills by providing best datasets feeding. Efficiency check will be comparatively better than existing systems. Only the userdefined datasets will be present. Current user cannot add any datasets. It is a module-based system in which multiple levels will be present. Datasets are fed only by the users. All those datasets will be proper and chances of having wrong datasets is less when compared to other applications. If datasets are at proper state, then efficiency mechanism of this application will be better. It contains five modules. Each module has six task actions. 


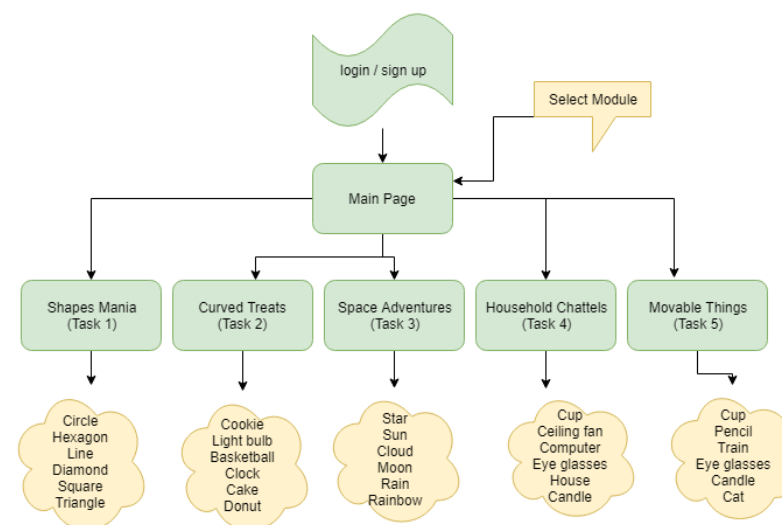

Figure 1: Modules Partition

This application will be multi grouped module-based action. It will be a chain application in which we need to keep on drawing the next entities. It consists of five modules. Each module consists of six drawing tasks. Therefore, totally 30 drawings tasks have to be completed in order to master basic single object drawing for beginners. This application will be the first stage for beginners.

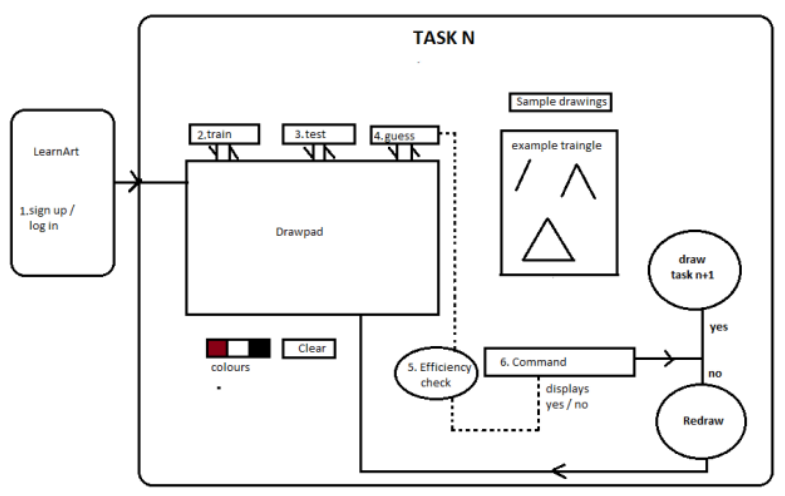

Figure 2: Architecture of workflow

It involves three main task actions: datasets alteration, training and testing of the drawings and checking the correctness of the drawing.

\section{MODULES}

\subsection{Datasets Alteration}

For each drawing, there will be predefined datasets. At least 1000 datasets of one entity is needed in order to get the correct result. The efficiency will be better in case of more datasets. The datasets obtained will be in numpy format and it has to be converted into binary format in order to lower the storage and fasten the efficiency check. Each dataset obtains 49 lines of binary code. Thus, overall 49000 lines of binary code will be present. If we are in need to correct the datasets, it can be altered by editing the lines of code.

\subsection{Training and Testing}

Training and testing sets has to be detached for evaluation purpose. Typically, when the data set separated into a training and testing set, $80 \%$ of dataset is used for training, and $20 \%$ of the dataset is used for testing. Analysis is aimlessly patterning the design to ensure that the training datasets and testing datasets are similar, using this similar data therefore it is understanding the characteristics of the model. it can diminish the effects of data discrepancies. By using the training set model is been processed by making predictions against the test dataset, since earlier the testing dataset contains the identified values for the characteristic that need to predict, so it is easier to justify whether the predictions of the model are precise.

\subsection{Checking the correctness}

When the given task action is drawn by the user, it checks the drawing with predefined datasets and produces the result whether it is right or wrong (see figure 3, 4 and 5).

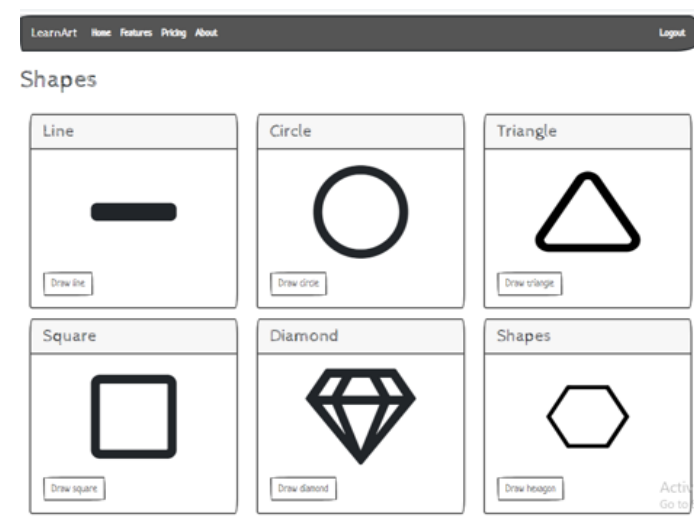

Figure 3: object selection

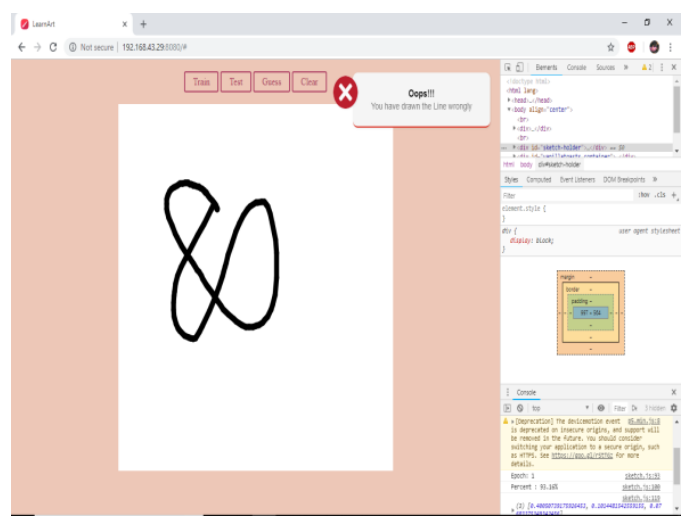

Figure 4: Analyzing wrong drawing

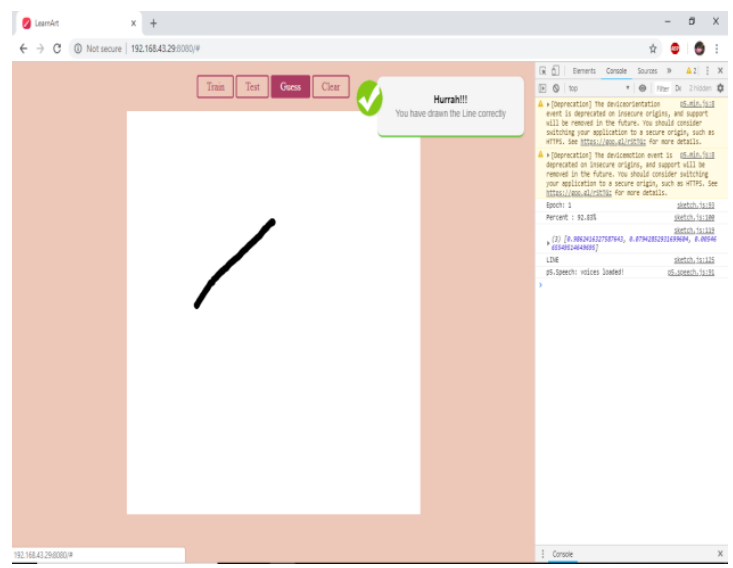

Figure 5: Analyzing correct drawing

Published By: 


\section{RESULTS \& ACCURACY OF TESTING}

Table 1: Overall Execution Parameters

\begin{tabular}{|c|c|c|c|}
\hline Module & Drawing & Percentage & Runtime \\
\hline 1 & Circle & $94.5 \%$ & 2 secs \\
\hline 2 & Clock & $87 \%$ & 5 secs \\
\hline 3 & Moon & $96 \%$ & 3 secs \\
\hline 4 & Candle & $91.5 \%$ & 2 secs \\
\hline 5 & Train & $94 \%$ & 3 secs \\
\hline
\end{tabular}

The Table1 shows each the example drawing of each module execution parameters. Percentage denotes the accuracy level check of each drawing. The maximum level of accuracy attained by each drawing. Runtime involves the time it took to check the correctness of the drawing. In this section, we cultivated the basic working of the proposed system that dually comes in the domain action of convolutional neural networks and weak artificial intelligence.

\section{CONCLUSION}

The proposed system admonishes the learners in a form of drawing, which composed of three task actions: datasets alteration, training and testing of the drawing and efficiency check. The prototype gives an advice in an effective manner to the learners who are interested in drawing based on the effectiveness of the prototype or the system was confirmed Though, it is essential to increase the accuracy of the model, also it should deal with a processing time. The training and testing of every task action consume certain time. But when more task actions are performed, it consumes some more amount of time. It can be adjusted using datasets alteration and by adding more datasets.

\section{REFERENCES}

1. Zhenyao Mo, J. P. Lewis and Ulrich Neumann, "SmartCanvas: a gesture-driven intelligent drawing desk system," January 2005, Proceedings of the 10th international conference on Intelligent user interfaces.

2. Colin G.Butler and Robert st.Amant, "HabilisDraw DT: A Bimanual Tool-Based Direct Manipulation Drawing Environment", April 2004, CHI '04 Extended Abstracts on Human Factors in Computing Systems.

3. Video sources from coding train and three brown one blue youtube videos.

4. Saeko Takagi, Noriyuki Matsuda, "A Learning Support System for Beginners in Pencil Drawing," February 2003, Proceedings of the 1st international conference on Computer graphics and interactive techniques in Australasia and South East Asia.

5. Tariq Rashid, "Make Your own neural network", Pages: 222 pages publisher: CreateSpace Independent Publishing Platform 2016-03-31

6. Umema Bohari, ting-u-chen, and vinayak, "To Draw or Not to Draw: Recognizing stroke-hover intent in Non-instrumental gesture-free mid-air sketching",March 2018 IUI '18: 23rd International Conference on Intelligent User Interfaces.

7. Wen wu and Yie yang, "Semi-Supervised Learning of Object Categories from PairedLocal Features “July 2008 CIVR '08: Proceedings of the 2008 international conference on Contentbased image and video retrieval. 